\title{
Impact of Integrating Computation into Undergraduate Curriculum: New Modules and Long-Term Trends
}

\section{Ms. Grace M. Lu, University of Illinois - Urbana-Champaign}

Grace Lu is a Ph.D. student and the computational teaching assistant in the Materials Science and Engineering department at the University of Illinois at Urbana-Champaign. She obtained her B.A. in Physics and Math from Northwestern University. Her research in the Trinkle Group uses machine learning and a variational principle to calculate mass transport in alloys.

\section{Prof. Dallas R Trinkle, University of Illinois at Urbana - Champaign}

Dallas R. Trinkle is a professor and associate head in Materials Science and Engineering at Univ. Illinois, Urbana-Champaign. He received his Ph.D. in Physics from Ohio State University in 2003. Following his time as a National Research Council postdoctoral researcher at the Air Force Research Laboratory, he joined the faculty of the Department of Materials Science and Engineering at Univ. Illinois, Urbana-Champaign in 2006. He was a TMS Young Leader International Scholar in 2008, received the NSF/CAREER award in 2009, the Xerox Award for Faculty Research at Illinois in 2011, the AIME Robert Lansing Hardy Award in 2014, co-chaired the 2011 Physical Metallurgy Gordon Research conference, and became a Willett Faculty Scholar at Illinois in 2015. His research focuses on defects in materials using density-functional theory, and novel techniques to understand problems in mechanical behavior and transport.

\section{Prof. Andre Schleife,}

André Schleife is a Blue Waters Assistant Professor in the Department of Materials Science and Engineering at the University of Illinois at Urbana-Champaign. He obtained his Diploma and Ph.D. at FriedrichSchiller-University in Jena, Germany for his theoretical work on transparent conducting oxides. Before he started at UIUC he worked as a Postdoctoral Researcher at Lawrence Livermore National Laboratory on a project that aimed at a description of non-adiabatic electron ion dynamics. His research revolves around excited electronic states and their dynamics in various materials using accurate computational methods and making use of modern super computers in order to understand, for instance, how light is absorbed in photo-voltaic materials.

\section{Dr. Cecilia Leal, University of Illinois at Urbana Champaign}

Cecília Leal is an Associate Professor in the Department of Materials Science and Engineering with affiliations at the Carle Illinois College of Medicine, the Materials Research Laboratory, and the Beckman Institute at the University of Illinois, Urbana-Champaign. She graduated in Industrial Chemistry from Coimbra University in Portugal and received her Ph.D. in physical chemistry from Lund University, supervised by Prof. Wennerström. After working for a year in the Norwegian Radium Hospital, she joined Prof. Safinya's Lab at the University of California in Santa Barbara as a postdoctoral fellow. Her research interests focus on the characterization and functionalization of lipid materials for cellular delivery. She is the recipient of a number of distinctions including the National Science Foundation CAREER award and the NIH New innovator award.

\section{Prof. Jessica Krogstad,}

Jessica A. Krogstad is an assistant professor in the Department of Material Science and Engineering at the University of Illinois, Urbana-Champaign. She received her PhD in Materials at the University of California, Santa Barbara in 2012. Between 2012 and 2014, she held a postdoctoral appointment in the Department of Mechanical Engineering at Johns Hopkins University. Her current research explores the interplay between phase or morphological evolution and material functionality in structural materials under extreme conditions. She also maintains interest in engineering education, specifically in outreach and design thinking. 


\section{Prof. Robert Maass, University of Illinois at Urbana - Champaign}

Robert Maass received a triple diploma in Materials Science and Engineering from the Institut National Polytechnique de Lorraine (INPL-EEIGM, France), Luleå Technical University (Sweden) and Saarland University (Germany) in 2005. In 2009, he obtained his PhD from the Materials Science Department at the École Polytechnique Fédérale de Lausanne (EPFL) in Switzerland. During his doctoral work, Robert designed and built an in-situ micro-compression set-up that he used to study small-scale plasticity with time-resolved Laue diffraction at the Swiss Light Source. From 2009-2011 he worked as a postdoctoral researcher at the Swiss Federal Institute of Technology (ETH Zurich) on plasticity of metallic glasses. Subsequently, he joined the California Institute of Technology as an Alexander von Humboldt postdoctoral scholar to continue his research on plasticity of metals. After working as a specialist management consultant for metals at McKinsey \& Co., he transferred to the University of Göttingen as a junior research group leader. He joined the faculty of the University of Illinois at Urbana-Champaign as Assistant Professor of Materials Science and Engineering in 2015.

\section{Dr. Pascal Bellon, University of Illinois at Urbana - Champaign}

After earning a PhD in Materials Science from University of Paris 6, France, Pascal Bellon worked during 7 years at CEA-Saclay, France, before joining the Department of Materials Science and Engineering at the University of Illinois at Urbana-Champaign as a tenure-track Assistant Professor in 1996, where he was promoted to the ranks of Associate Professor in 2002 and Full Professor in 2009. He received an NSF career award in 1998 and awards from the Academy for Excellence in Engineering Education from the University of Illinois in 1998, 1999 and 2000. He received the Don Burnett teaching award in 2000, the Accenture Engineering council award for Excellence in Advising in 2007 and the Stanley Pierce award in 2009. In 2012 Pascal Bellon was named a Racheff faculty scholar, and in 2016 he was inducted as the Donald W. Hamer Professor in Materials Science and Engineering.

\section{Pinshane Y Huang, University of Illinois at Urbana-Champaign}

Pinshane Y. Huang is an Assistant Professor in the Department of Materials Science and Engineering at the University of Illinois, Urbana-Champaign. She holds a Ph.D. in Applied and Engineering Physics from Cornell University, as well as a B.A. in Physics from Carleton College.

Prof. Nicola H Perry, Department of Materials Science \& Engineering, University of Illinois at UrbanaChampaign

Prof. Matthew West, University of Illinois at Urbana - Champaign

Matthew West is an Associate Professor in the Department of Mechanical Science and Engineering at the University of Illinois at Urbana-Champaign. Prior to joining Illinois he was on the faculties of the Department of Aeronautics and Astronautics at Stanford University and the Department of Mathematics at the University of California, Davis. Prof. West holds a Ph.D. in Control and Dynamical Systems from the California Institute of Technology and a B.Sc. in Pure and Applied Mathematics from the University of Western Australia. His research is in the field of scientific computing and numerical analysis, where he works on computational algorithms for simulating complex stochastic systems such as atmospheric aerosols and feedback control. Prof. West is the recipient of the NSF CAREER award and is a University of Illinois Distinguished Teacher-Scholar and College of Engineering Education Innovation Fellow.

\section{Prof. Timothy Bretl}

Timothy Bretl is an Associate Professor of Aerospace Engineering at the University of Illinois at UrbanaChampaign. He received his B.S. in Engineering and B.A. in Mathematics from Swarthmore College in 1999, and his M.S. in 2000 and Ph.D. in 2005 both in Aeronautics and Astronautics from Stanford University. Subsequently, he was a Postdoctoral Fellow in the Department of Computer Science, also at Stanford University. He has been with the Department of Aerospace Engineering at Illinois since 2006, where he now serves as Associate Head for Undergraduate Programs. He holds an affiliate appointment in the Coordinated Science Laboratory, where he leads a research group that works on a diverse set of 
projects (http://bretl.csl.illinois.edu/). Dr. Bretl received the National Science Foundation Early Career Development Award in 2010. He has also received numerous awards for undergraduate teaching in the area of dynamics and control, including all three teaching awards given by the College of Engineering at Illinois (the Rose Award for Teaching Excellence, the Everitt Award for Teaching Excellence, and the Collins Award for Innovative Teaching).

\section{Dr. Geoffrey L Herman, University of Illinois at Urbana - Champaign}

Dr. Geoffrey L. Herman is a teaching associate professor with the Deprartment of Computer Science at the University of Illinois at Urbana-Champaign. He also has a courtesy appointment as a research assistant professor with the Department of Curriculum \& Instruction. He earned his Ph.D. in Electrical and Computer Engineering from the University of Illinois at Urbana-Champaign as a Mavis Future Faculty Fellow and conducted postdoctoral research with Ruth Streveler in the School of Engineering Education at Purdue University. His research interests include creating systems for sustainable improvement in engineering education, conceptual change and development in engineering students, and change in faculty beliefs about teaching and learning. 


\title{
Impact of Integrating Computation into Undergraduate Curriculum: New Modules and Long-Term Trends
}

\begin{abstract}
Computational methods have become increasingly used in both academia and industry. At the University of Illinois Urbana Champaign, the Department of Materials Science and Engineering (MSE), as part of a university-funded educational innovation program, has integrated computation throughout its undergraduate courses since 2014. Within this curriculum, students are asked to solve practical problems related to their coursework using computational tools in all required courses and some electives. Partly in response to feedback from students, we have expanded our current curriculum to include more computational modules. A computational module was added to the freshman Introduction to Materials Science and Engineering class; thus, students will be expected to use computational tools from their first year onwards. In this paper, we survey students who are currently taking courses with integrated computation to explore the effects of gradually introducing students to programming as well as both macro- and micro-scale simulations over multiple years. We investigate the improving confidence level of students, their attitude towards computational tools, and their satisfaction with our curriculum reform. We also updated our survey to be more detailed and consistent between classes to aid in further improvements of our MSE curriculum.
\end{abstract}

\section{Introduction}

Computational tools have become indispensable for materials science both in research and industry. According to the 2009 survey by Thornton et al. ${ }^{1}$, and the 2018 followup by Enrique et al. ${ }^{2}$, employers that hire Materials Science and Engineering (MSE) students value computational materials science education and desire 50\% of their hires to have a computational MSE background . Additionally, they believe MSE graduates should at least be aware of what simulations used in their field and what is computationally feasible ${ }^{2}$. They also highlighted a gap between industry and what is taught in class - while students are typically taught molecular and atomic-scale simulations in class, employers are more interested in macroscopic, continuum models ${ }^{1}$.

As a result of these surveys, faculty started an initiative project to address the shortcomings in our MSE curriculum. This project, under a Strategic Instructional Initiatives Program (SIIP) of the College of Engineering, seeks to establish a collaborative environment to incorporate computational modules into classes and enhance instruction. This was inspired by Henderson et al. ${ }^{3,4,5,6}$, and more information can be found in our earlier publications ${ }^{7,8,9,10,11}$. 
Our previous studies measure the effectiveness of our computational curriculum reform. Mansbach et al. ${ }^{8}$ found that students' grades and performance on exams improved after adding computation to their coursework. Zhang et al. ${ }^{10}$ surveyed students and found that they desire earlier and more computational content, preferably starting in their first year of instruction. Followup surveys by Lee et al. ${ }^{11}$ found that graduating students became comfortable with using computational tools to solve MSE problems and more than half of students believed that the addition of computation helped them understand the course content.

In this paper, we first describe our curriculum and the new computational modules we have developed and integrated into our classes. To measure the effectiveness of the new computational modules, we investigate both the comfort of students with using the computational tools and how relevant the module is to the course. Given the maturing of our curriculum, we then compare survey results to investigate the temporal trends of students' comfort with the computational tools, their opinions on computation in general, and their satisfaction with the current MSE curriculum.

\section{Curriculum and Computational Modules}

The courses involved in the curriculum reform include all required undergraduate courses and some semi-required and elective courses as listed in Table 1. Each course has two computational modules, except for MSE 182 (1), MSE 422 (1), and MSE 406 (3). These modules cover content directly applicable to the course, as listed in Table 2 . This is the first year where there has been a module focused on the use of visualization software, such as OVITO. The specific tools that are used in the computational modules are:

- OVITO $^{12}$ for atomic visualization (first year using this software)

- OOF $2^{13}$, using the nanohub.org ${ }^{14}$ interface, for finite element methods (FEM)

- MATLAB ${ }^{15}$ for scientific computing e.g. fitting data and solving differential equations

- LAMMPS ${ }^{16}$ for molecular dynamics (MD)

- ThermoCalc ${ }^{17}$ (CALPHAD) for computing phase diagrams

- Quantum Espresso ${ }^{18}$ for density-functional theory (DFT) calculations

While the curriculum has mostly stayed constant and is described in earlier work ${ }^{7,10}$, two additional modules have been developed over the last two years for classes, MSE 182 and MSE 422, that had previously lacked computation. Using OVITO, since Fall 2019, students in MSE 182 were asked to visualize the diamond cubic structure of $\mathrm{Si}$ and sketch various planes. Then, they compared the OVITO image to real STEM images and labeled corresponding columns of atoms. After that, they created an animation showing a Si supercell rotating 360 degrees. While there are no other modules focused on OVITO, students are expected to use OVITO or other visualization software, e.g. to analyze their MD results. Thus, students will use the skills from this module again later in the curriculum. This also marks the first computational module added 
Table 1: All MSE classes involved in this paper. Semi-required classes are required for some focus areas. MSE 404 is the general course number for Laboratory Studies in MSE, but in this paper, will only refer to Computation in MSE.

\begin{tabular}{c|l|c|c} 
Number & Name & Level & Type \\
\hline 182 & Introduction to MatSE & Freshman & Required \\
201 & Phase and Phase Relations & Sophomore & Required \\
206 & Mechanics for MSE & Sophomore & Required \\
401 & Thermodynamics of Materials & Junior & Required \\
402 & Kinetic Processes in Materials & Junior & Required \\
406 & Thermal and Mechanical Behavior of Materials & Junior & Required \\
304 & Electronic Properties of Materials & Junior & Semi-required \\
$404^{*}$ & Laboratory Studies in MSE: Computation in MSE & Senior & Semi-required \\
422 & Electrical Ceramics & Junior/Senior & Elective \\
440 & Mechanical Behaviors of Materials & Junior/Senior & Elective
\end{tabular}

to a freshman course, so students will now be exposed to computational tools from the first year of their undergraduate curriculum.

Table 2: All computational modules in SIIP classes. The classes marked with * have computational modules which were added after 2017.

\begin{tabular}{c|c|c|c|c|c|c} 
Course & Visualization & FEM & MATLAB & MD & CALPHAD & DFT \\
\hline $182^{*}$ & $\mathrm{X}$ & & & & $\mathrm{X}$ & $\mathrm{X}$ \\
201 & & & & & $\mathrm{X}$ & \\
206 & & $\mathrm{X}$ & $\mathrm{X}$ & & & $\mathrm{X}$ \\
304 & & & & $\mathrm{X}$ & $\mathrm{X}$ & \\
401 & & & & $\mathrm{X}$ & & $\mathrm{X}$ \\
402 & & $\mathrm{X}$ & $\mathrm{X}$ & $\mathrm{X}$ & $\mathrm{X}$ & $\mathrm{X}$ \\
404 & & $\mathrm{X}$ & & $\mathrm{X}$ & & \\
406 & & & & $\mathrm{X}$ & & \\
$422^{*}$ & & $\mathrm{X}$ & $\mathrm{X}$ & & &
\end{tabular}

Since Spring 2019, there has also been a module in MSE 422, which uses LAMMPS to explore the ionic conductivity of $\mathrm{CeO}_{2}$. Using classical force fields, they first calculated the defect formation energy of an oxygen vacancy and used that to calculate the equilibrium vacancy concentration. Next, they calculated the diffusion coefficient, estimated the ionic conductivity, and finally, calculated the activation energy for diffusion.

\section{Expanded Survey}

To help us with our curriculum reform, we have updated the pre- and post-course surveys to pinpoint areas of improvement in the current computational curriculum. These questions seek to 
measure the performance of the computational modules both to increase student comfort with computational tools and to aid students' understanding of related course material in their classes. We hope to use the results of these surveys to improve integration of computational modules and pinpoint weaknesses. The current questions are:

- Do you think computational tools are important for materials science and engineering? (Very Important - 12345 - Very Unimportant)

- Do you think computational materials science skills are important for your post-graduation career?

(Very Important - 12345 - Very Unimportant)

- In general, do you think the computational experiences you have had in MatSE classes are beneficial to you?

(Very Beneficial - 12345 - Not Beneficial at All)

- Should there be more or less computational material in MatSE classes?

(Much More - 12345 - Much Less)

- Which of the following computational methods do you think is the most difficult one?

- What do you think is the best time to start learning computational skills in MatSE classes?

- Do you think that Computational Module _- is relevant to the course syllabus? (This question will be asked for each computational module in the class.)

(Very Relevant - 12345 - Not Relevant at All)

- Do you think the computational modules help you understand the related course materials in your class?

(Very Beneficial - 12345 - Not Beneficial at All)

- Do you understand the objective of the computational modules?

(Understand Very Well - 12345 - Don't Understand Very Well)

Furthermore, to reduce bias, we have made questions that measure student comfort with computational modules more detailed. Instead of asking whether or not students are familiar with various computational tools, we will now ask students about their comfort in applying the computational tools to specific class-related problems.

- If you were asked to visualize and compare different crystal structures, how comfortable would you be using the following approaches?

Draw (pencil and paper)

Ovito (or a similar software)

(Very comfortable - 12345 - Very uncomfortable)

(Very comfortable - 12345 - Very uncomfortable)

These new questions will be standardized across all classes and asked at the beginning and end of the semester. The results will then be used for the further development of new modules and improvements of the existing ones. 


\section{Effectiveness of New Modules}

To measure the effectiveness of the new modules, we surveyed students at the end of the semester to measure their perceived comfort levels with the computational tools, and if the modules were useful when placed in context with the class as a whole. For MSE 182, we asked students to answer the following three questions:

- Q1: If you were asked to visualize and compare different crystal structures, how comfortable would you be using OVITO or similar software? (Very comfortable - 12345 - Very uncomfortable)

- Q2: Do you think the computational modules help you understand the related course materials in MatSE 182?

(Strongly agree - $12345-$ Strongly disagree)

- Q3: Do you understand the objective of the computational modules?

(Strongly agree - 12345 - Strongly disagree)

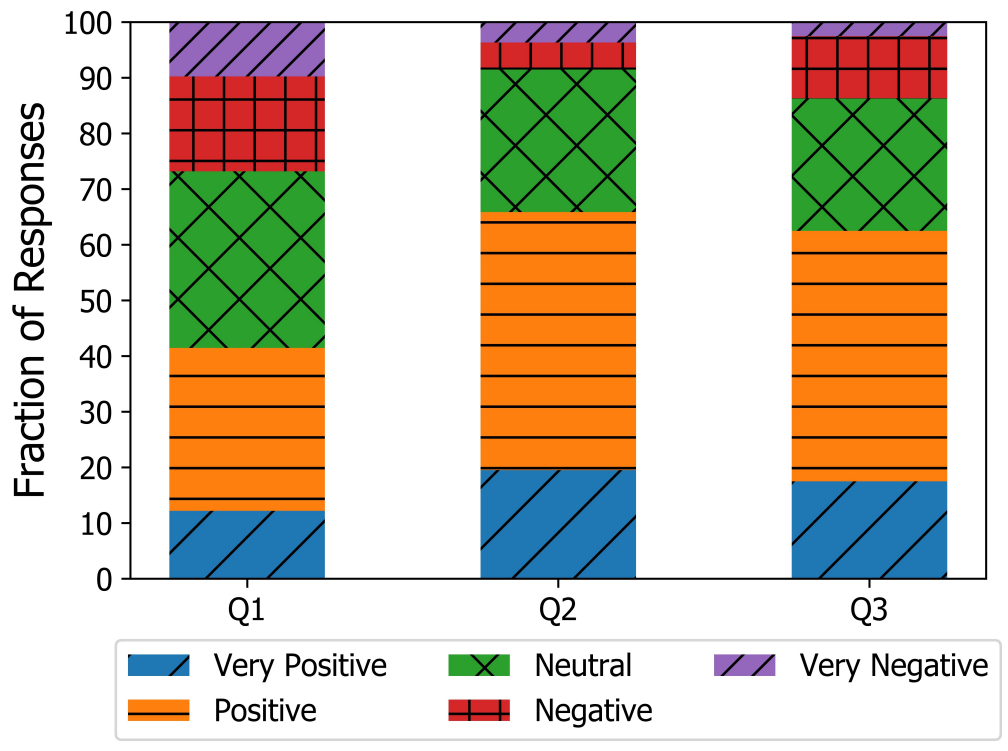

Figure 1: Students' feedback on the computational module in MSE 182. There were 82 responses to this survey, which was taken at the end of the semester.

Their responses are shown in Fig. 1. While Lee et al. ${ }^{11}$ found that only a third of graduating seniors believed that the computational modules were helpful in understanding the course material, here, $66 \%$ ( $p$-value $<10^{-6}$ ) of students believed that to be the case. Results may be skewed by relevancy because graduating seniors were years separated from modules taken in their sophomore and junior years while this survey was taken at the end of the semester. Additionally, $41 \%$ of students were comfortable (chose either option 1 or 2) using OVITO to visualize crystal structures. Because this module is the first one focused on OVITO, it is unclear if students have become more comfortable after the class or not. More open-ended problems given to the students, for them to explore this tool on their own may be needed to alleviate this. 
For MSE 422, we asked students to answer the following three questions:

- Q1: You are comfortable with explaining MD to your classmates. (Strongly agree - 12345 - Strongly disagree)

- Q2: You are comfortable with conducting a project that requires usage of MD in your future career.

(Strongly agree - 12345 - Strongly disagree)

- Q3: You think the computational module in 422 (lecture plus homework) is relevant to the course syllabus.

(Strongly agree - 12345 - Strongly disagree)



Figure 2: Students' feedback on the computational module in MSE 422. There were 21 responses to the survey.

Their responses in Fig. 2 show that after taking the class, more than $25 \%$ of students were comfortable explaining MD to their classmates, and less than $20 \%$ of students were comfortable using MD in a future project. These results are in agreement with Lee et al. ${ }^{11}$ who compared the confidence of sophomores and seniors and found that while sophomores had $0 \%$ confidence in using MD, almost $50 \%$ of graduating seniors were confident. We therefore conclude that students become more confident in MD as they are exposed to it more frequently between the start and end of the MSE curriculum. Due to graduate student enrollment in MSE 422 and flexible scheduling of junior-level electives, it is difficult to draw further conclusions about their comfort as they progress through the curriculum. Additionally, $50 \%$ of students thought that the content in the computational module was relevant to the course, which is larger than the $33 \%$ reported by Lee et. al's ${ }^{11}$ survey of graduating seniors across the entire curriculum. Using their feedback, we can conclude that the new computational module was well-integrated into the curriculum. 


\section{Temporal Trends}

Our previous surveys show the increasing confidence in using computational tools between both the entry and exit surveys, given at the start and end of their SIIP courses ${ }^{7,10,11}$ and more broadly, between the entry survey of MSE 201 and in their senior year ${ }^{11}$. We also found that senior students are the most likely to want to start learning computation tools in their freshman year, but students are generally satisfied with the current amount of computation in their classes ${ }^{11}$.

To expand our understanding of the long-term effects of our curriculum reform, we measure how students' opinions toward computational tools have changed throughout the years. We compare surveys from 2016-2020 and split the results into three sections: (1) students' comfort with using computational methods, (2) students' general attitude toward computational tools, and (3) students' satisfaction with the amount of computational modules in the MSE curriculum.

\subsection{Comfort with Computational Tools}

Students were asked several survey questions to rate their comfort in using a variety of computational methods to perform representative calculations from their course. To measure their comfort with FEM methods, students were asked one of the following questions:

- MSE 206: If you were asked to determine the bending of a beam under loads, how comfortable would you be using Finite Element (e.g. OOF2)?

(Very comfortable - 12345 - Very uncomfortable)

- MSE 406: If you were asked to determine the stress field ahead of a crack tip, how comfortable would you be using Finite Element (e.g., OOF2)?

(Very comfortable - 12345 - Very uncomfortable)

Figure 3 shows the students' comfort with using FEM in MSE 206 and MSE 406 in 2016, 2017, 2018, and 2019. In two years, we see that the percentage of students who are are least neutral (who select from options 1-3) towards using FEM in MSE 206 increases slightly from $28 \%$ to $33 \%$ ( $p$-value $=0.25$ ). Because this is the first class where students are exposed to FEM, changes in comfort levels are expected to be small. More strikingly, the percentage of students who are very uncomfortable drops drastically from 59\% to $34 \%\left(p\right.$-value $\left.=1.7 * 10^{-4}\right)$. Given that MSE 206 is the first class where students are exposed to FEM, these results imply that as our curricular changes are becoming more mature, students are perceiving themselves as more confident with computational tools without any further exposure. Even though the module itself has not changed throughout the years, this change could be a result of additional experience with emphasizing connections with the coursework and greater understanding of common student pitfalls, leading to updated instructions. The low statistical significance may be a result of the instructor and the teaching assistant for the course changing throughout the years, so no instructor has four years of experience.

For MSE 406, although the number of students who are at least neutral drops from 65\% in 2016 to $42 \%$ ( $p$-value $\left.=1.0 * 10^{-4}\right)$ in 2018 , it rises back up to $73 \%$ ( $p$-value $\left.>0.25\right)$ in 2019 . This can be explained by looking at the scores for the first midterm exam as shown in Table 3. (We are using midterm scores rather than final grades because the grades were scaled so that the distribution would be similar for different years.) While the questions asked have not 


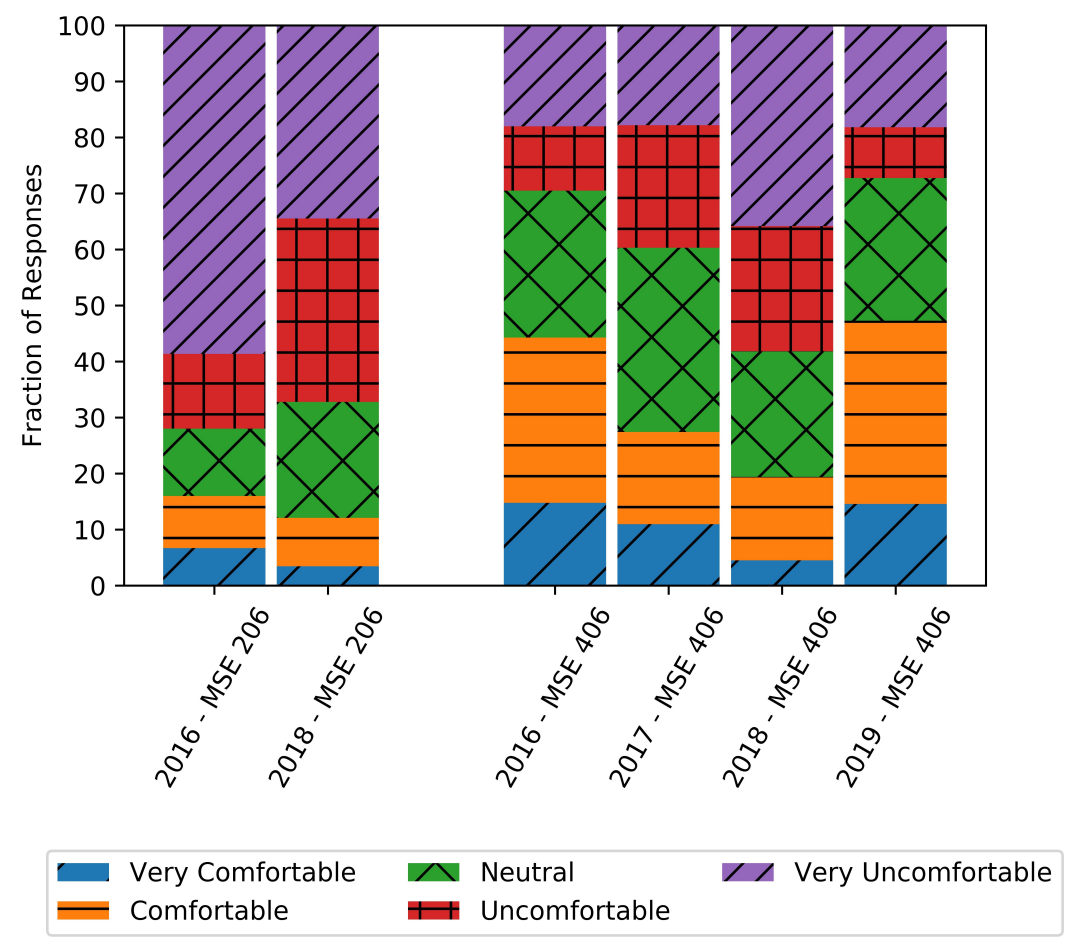

Figure 3: Students' comfort level in MSE 206 and MSE 406 over time. Data from 2016 is from Kononov et $\mathrm{al}^{7}$. From left to right, there are 75, 58, 70, 76, 67, and 55 students surveyed for each class. There is graduate student enrollment and survey responses in 400-level classes.

fundamentally changed, there is a noticeable decline in score and an increased standard deviation between 2016 and 2018. This implies that students' performance became more heterogeneous, so there were many students in those years who had low scores in the class and therefore low confidence levels in their overall class performance. This lower confidence in the class as a whole may have led to lower confidence in using the computational tools. Because the survey is taken anonymously, we cannot directly measure correlation between students who performed more poorly in the class and their perceived confidence in using computational tools. The low statistical significance between 2016 and 2019 is due to two competing processes: slightly lower scores and increased experience with teaching the class with the incorporated computational modules.

Table 3: Midterm Grades in MSE 406 from 2016 and 2019.

\begin{tabular}{c|c|c} 
Year & Mean Score & Standard Deviation \\
\hline 2016 & 77 & 13 \\
2017 & 65 & 18 \\
2018 & 59 & 16 \\
2019 & 71 & 15
\end{tabular}




\subsection{Attitude Towards Computational Tools}

To understand students' attitudes toward computational tools, in general, we asked the following question:

- I think computational materials science skills are important for my post-graduation career. (Strongly Agree - 12345 - Strongly Disagree)

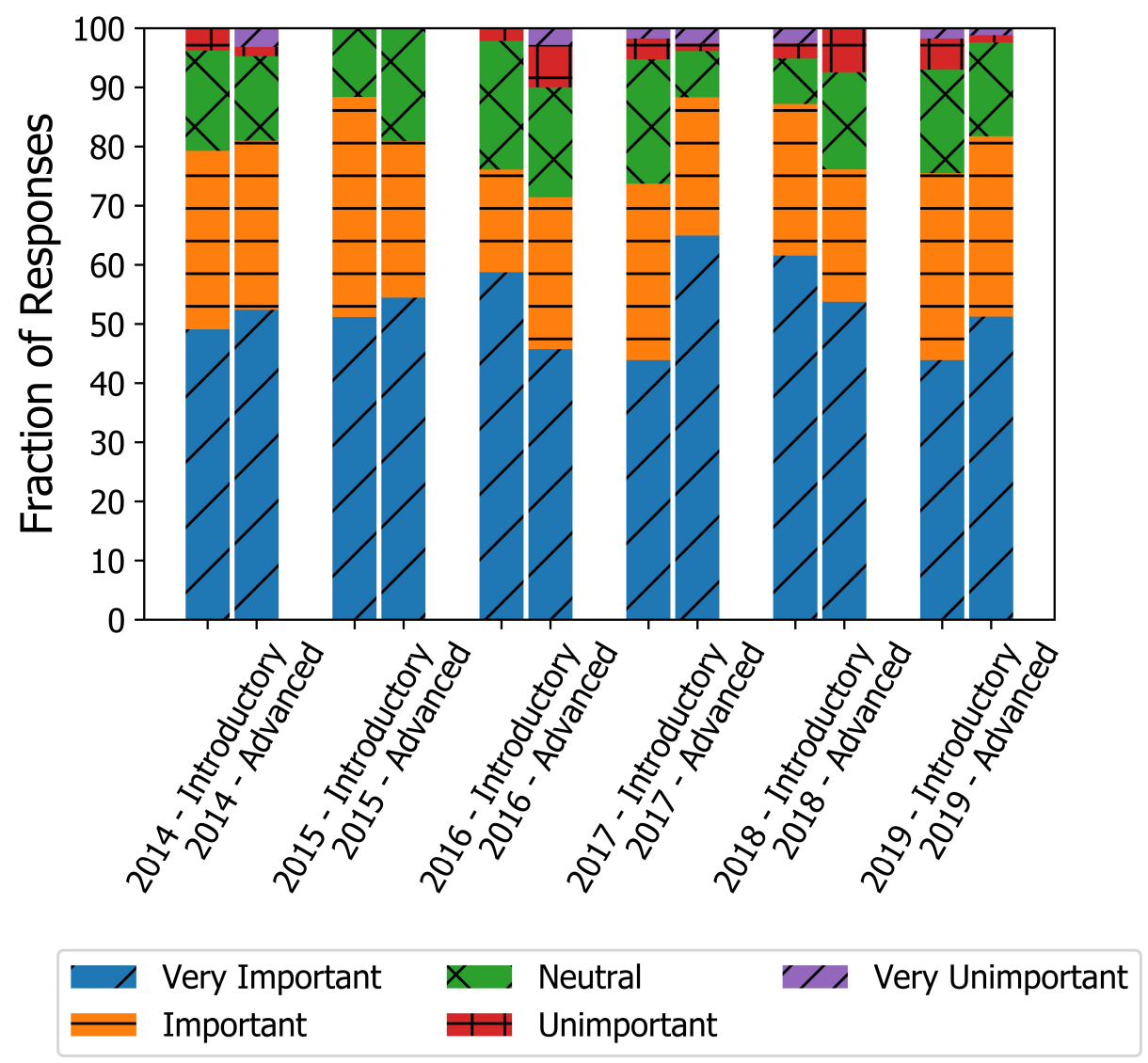

Figure 4: Students' attitudes towards computational materials science skills from 2014-2019. Data from 2014-2016 is from Kononov et al. ${ }^{7}$, and data from 2017 is from Zhang et al. ${ }^{10}$. There are 53, $63,43,68,46,70,57,77,39,67,57$, and 82 students, per category, from left to right.

Figure 4 shows the students' responses split between introductory and advanced classes. Introductory-level classes are defined as 100 and 200 level classes, while advanced classes are 300 and 400 level classes. We see that students continue to believe that computational skills are important after graduation with no temporal trends. $80 \%$ of students in 2014 believe that computational methods are important (chose option 1 or 2 ) to their post-graduate careers compared to $79 \%$ of students in 2019. Similarly, their attitudes do not change between introductory and advanced classes. Across the six years, $80 \%$ of students in both introductory and advanced classes believed that computational tools were important for their post-graduation careers. Since students enrolled in advanced classes, excluding the graduate students or transfer students, have already taken other courses with computational modules, our results imply that 
further exposure to computational tools, which come with step-by-step instructions on how to use the software and focus on solving straightforward, practical problems, does not impact students' perception of the importance of these tools for their post-graduate career. The modules, therefore, may benefit from more open-ended exploration of the software so that students can get a greater understanding of the limits of the computational tools.

\subsection{Satisfaction with Computational Modules in Curriculum}

We also surveyed students on their satisfaction with the amount of computation in the MSE curriculum. To do so, we asked students the following question:

- I would like to use computation in my MatSE classes... (Much More - 12345 - Much Less)

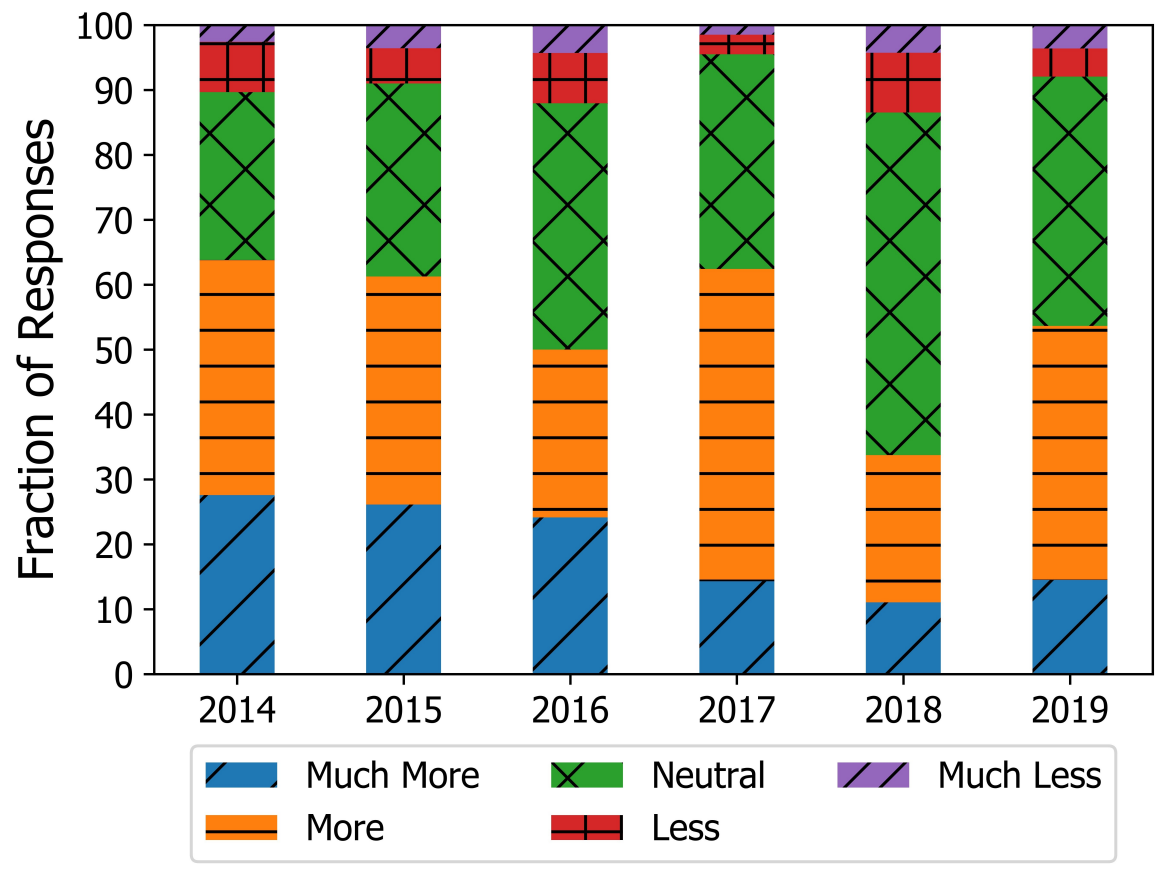

Figure 5: Students' attitudes towards the amount of computation in MSE classes from 2014-2019. Data from 2014-2016 is from Kononov et al. ${ }^{7}$, data from 2017 is from Zhang et al. ${ }^{10}$, and data from 2018 is from Lee et al. ${ }^{11}$. There are 116, 111, 116, 133, 163, and 138 students surveyed each year, ordered from left to right.

Once again, we trace students' responses from 2014-2019 shown in Figure 5. We see that students are generally satisfied with the amount of computation in their course with $38 \%$ of students in 2019 responding that they thought that the amount of computation already implemented into their classes was suitable, and less than $10 \%$ of students wanting a decrease in the amount of computation. Temporally, the number of students who want more computation (chose option 1 or 2) in their coursework decreases from 64\% in 2014 to $34 \%$ in 2018 ( $p$-value < $\left.10^{-6}\right)$ and $54 \%$ in $2019\left(p\right.$-value $\left.=7.8 * 10^{-3}\right)$. The increase between 2018 and 2019 may have occurred due to the new inclusion of freshmen in the survey. The freshmen have only been 
exposed to one module in MSE 182 rather than the two in MSE 201, which had previously been the first course with integrated computational tools in the MSE curriculum. We conclude that exposure to the computational modules is fulfilling students' desire for more computation in their MSE classes, but that more modules are still desired. We are exploring whether modules can be added to more MSE classes and plan to expand the number of modules in MSE 182 and MSE 422, which currently only have one.

\section{Conclusion}

Since the survey of employers of MSE students by Thornton et. al. ${ }^{1}$, our department has integrated computational modules into core classes. In response to feedback by students who have wanted more and earlier computation in their classes, we have added new modules in MSE 422 and MSE 182, which now integrates freshmen into our curricular reform.

Now that computational modules have been included for many years, we explored the long-term temporal trends of student confidence with computational tools, their opinions about computational modules in general, and their satisfaction with the amount of computation in their curriculum. As the curriculum is becoming more mature, we found that students are becoming more comfortable with computational tools without additional exposure. Because we are only surveying students at the end of the semester, feedback is collected weeks after the modules have been completed. To more accurately measure students' comfort levels, feedback during and directly after computational modules should be collected. Furthermore, we are only measuring students' perception of their comfort levels with the computational modules, and not their comfort levels directly.

Students have continued to believe that computational skills are important for their post-graduation career, but surveys of alumni will be needed to directly measure any impact. Additionally, because our results rely solely on surveys, measuring the direct impact of the computational tools on student learning will necessitate a more thorough analysis of students' performance in their classes. Furthermore, students continue to desire more integrated computation in their coursework, so more modules will need to be developed. To aid in this process, we have expanded our current survey to further probe the performance of our computational modules, and we will report on the results once they are available.

\section{Acknowledgements}

This work received IRB approval under protocol number 14094. This work was supported by the College of Engineering and the Department of Materials Science and Engineering at the University of Illinois at Urbana-Champaign as part of the Strategic Instructional Initiatives Program (SIIP), by a National Science Foundation CAREER Award to J.A.K. (Grant No. 1654182), by a NSF CAREER Award to P.H. (Grand No. DMR-1846206) by a NSF CAREER Award to R.M. (Grant No. 1654065), by a NSF CAREER Award to C.L. (Grant No. DMR-1554435), by a NSF CAREER Award to A.S. (Grant No. DMR-1555153), a NSF Grant to N.H.P (Grant No. DMR-1945482), and by a NSF Grant to P.B. (Grant No. DMR-1709857). This material is based upon work supported by the National Science Foundation Graduate Research Fellowship under Grant No. 1746047. Any opinions, findings, and conclusions or 
recommendations expressed in this material are those of the authors and do not necessarily reflect the views of the National Science Foundation.

\section{References}

[1] K. Thornton, S. Nola, R. Edwin Garcia, M. Asta, and G.B. Olson. Computational materials science and engineering education: A survey of trends and needs. JOM, 61(10):12-17, 2009.

[2] R.A. Enrique, K. Thornton, and M. Asta. Computational materials science and engineering education: An updated survey of trends and needs. JOM, 70(9):1644-1651, 2018.

[3] Maura Borrego and Charles Henderson. Increasing the use of evidence-based teaching in stem higher education: A comparison of eight change strategies. Journal of Engineering Education, 103(2):220 - 252, 2014.

[4] Charles Henderson and Melissa H. Dancy. Barriers to the use of research-based instructional strategies: The influence of both individual and situational characteristics. Phys. Rev. ST Phys. Educ. Res., 3(2):020102, 2007.

[5] Charles Henderson, Andrea Beach, and Noah Finkelstein. Facilitating change in undergraduate stem instructional practices: An analytic review of the literature. Journal of Research in Science Teaching, 48(8):952 $-984,2011$.

[6] Charles Henderson, Melissa Dancy, and Magdalena Niewiadomska-Bugaj. Use of research-based instructional strategies in introductory physics: Where do faculty leave the innovation-decision process?. Phys. Rev. ST Phys. Educ. Res., 8(2):020104, 2012.

[7] Alina Kononov et al. Computational curriculum for matse undergraduates. In 2017 ASEE Annual Conference \& Exposition, Columbus, Ohio, June 2017. ASEE Conferences.

[8] Rachael Alexandra Mansbach et al. Work in progress: Computational modules for the matse undergraduate curriculum. In 2016 ASEE Annual Conference \& Exposition. ASEE Conferences.

[9] R. Mansbach, A. Ferguson, K. Kilian, J. Krogstad, C. Leal, A. Schleife, D. R. Trinkle, M. West, and G. L. Herman. Reforming an undergraduate materials science curriculum with computational modules. "J Mater Educ, 38(3-4):161 - 174, 2016.

[10] Xiao Zhang et al. Computational curriculum for matse undergraduates and the influence on senior classes. In 2018 ASEE Annual Conference \& Exposition, 2018.

[11] Cheng-Wei Lee et al. Impact of computational curricular reform on non-participating undergraduate courses: Student and faculty perspective. In 2019 ASEE Annual Conference \& Exposition, 2019.

[12] A. Stukowski. Visualization and analysis of atomistic simulation data with ovito-the open visualization tool. Modelling and Simulation in Materials Science and Engineering, 18(1), 2010.

[13] A.C.E. Reid, S.A. Langer, R.E. Lua, R.C.and García, and V.R. Coffman. Modelling microstructures with oof2. International Journal of Materials and Product Technology, 35, 2009.

[14] Stephen Langer, R. Edwin García, and Andrew Reid. Oof2, Nov 2007. URL https://nanohub.org/resources/oof2.

[15] MATLAB version 8.5.0.197613 (R2015a). The Mathworks, Inc., Natick, Massachusetts, 2015.

[16] S. Plimpton. Fast parallel algorithms for short-range molecular dynamics. Journal of Computational Physics, 117(1):1-19, 1995. ISSN 00219991. 
[17] J.-O. Andersson, T. Helander, L. Höglund, P. Shi, and B. Sundman. Thermo-calc dictra, computational tools for materials science. Calphad: Computer Coupling of Phase Diagrams and Thermochemistry, 26(2):273-312, 2002.

[18] Paolo Giannozzi et al. Quantum espresso: a modular and open-source software project for quantum simulations of materials. Journal of Physics: Condensed Matter, 21(39):395502 (19pp), 2009. URL http://www. quantum-espresso.org. 